\title{
Estreptococo do grupo B: rastrear ou não rastrear no Brasil? Eis a questão
}

\author{
Group B streptococcus: to screen or not to screen in Brazil? That is the question
}

\author{
Eliana Amaral*
}

Os estudos sobre a prevalência da colonização pelo estreptococo do grupo B (EGB) refletem a preocupação dos grupos de pesquisa nacionais com as complicações associadas a este agente, quando há intervenções eficazes para seu controle. Em artigos publicados na RBGO, sendo um neste fascículo, foram avaliadas as prevalências da colonização pelo EGB entre gestantes com mais de 35 semanas realizando pré-natal em hospitais-escola do Paraná e de Santa Catarina ${ }^{1,2}$. Os dois estudos coletaram amostras anal e vaginal e utilizaram meio específico para isolamento e identificação da bactéria, conforme recomendação internacional (Schrag et al., 2002) ${ }^{3}$. As prevalências encontradas foram 14,9 e $21,6 \%$, respectivamente, valores que estão dentro da variação relatada em outros países.

O EGB está associado a infecção materna puerperal. Mas o grande temor é a sepse neonatal precoce, que se observa com menor freqüência, porém com maior mortalidade entre prematuros. A colonização ocorre em metade dos recém-nascidos de mães portadoras, mas apenas $2 \%$ terão os quadros infecciosos neonatais. Se a prevalência de colonização materna nacional for de $20 \% \mathrm{em}$ média, devemos ter uma incidência de dois casos para cada 1000 nascidos vivos na ausência de intervenções profiláticas. Não está implementada a vigilância de casos de sepse neonatal no país, nem existem dados populacionais que nos permitam confirmar esta incidência.

O uso de antibiótico no trabalho de parto (penicilina intra-venosa) é a intervenção profilática recomendada ${ }^{4}$. A polêmica está na estratégia mais adequada para selecionar as gestantes que devem receber esta profilaxia, visto que apenas dois em cada 1000 partos ou $1 \%$ das gestantes colonizadas pelo EGB podem ter recém-nascidos acometidos pela sepse neonatal precoce, como vimos. A primeira estratégia proposta e adotada internacionalmente foi o uso de fatores de risco que incluem: parto prematuro, temperatura superior a $>38^{\circ} \mathrm{C}$ durante trabalho de parto, ruptura de membranas por mais de 18 horas, ou antecedente de feto anterior acometido por EGB e infecção urinária por EGB na gestação.

O debate sobre o uso de cultura pré-natal tardia em substituição aos fatores de risco é intenso. A mudança de conduta nos EUA seguiu as recomendações do Centers for Disease Control and Prevention (CDC). Em 1996, propunham-se as duas estratégias, fatores de risco no trabalho de parto ou triagem pré-natal, para orientar a profilaxia intra-parto. Na publicação de 2002, decidiu-se pela exclusão da primeira estratégia, visto que metade dos casos de sepse precoce ocorria sem fatores de risco e que se conseguia uma melhor adesão à profilaxia pelos profissionais quando o resultado da cultura era disponivel. Outras razões para uso da triagem incluíram o argumento de reduzir o uso de antibiótico desnecessário em mulheres não colonizadas, facilitar a recomendação e a definição de indicadores para avaliação da implementação da estratégia. Entretanto, as diferentes estratégias não diferiram quanto ao percentual estimado de uso de antibiótico intraparto, cerca de $24 \%$, com seus riscos potenciais que são a anafilaxia pela penicilina e desenvolvimento de infecção neonatal e materna com bactérias resistentes (Schrag et al., 2002) . $^{3}$

No Brasil, não há nenhum consenso ou recomendação técnica sobre o tema. No projeto Diretrizes da Associação Médica Brasileira ${ }^{5}$, sugere-se realização de cultura no terceiro trimestre se houver fatores de risco, proposta diferente das que constam na literatura internacional. Nos eventos científicos e publicações de obstetras, neonatologistas e infectologistas observa-se o interesse crescente pelo tema. A preocupação de quantificar inicialmente o problema, como o fizeram Beraldo et al. ${ }^{1}$ e Pogere et al. ${ }^{2}$, é louvável e necessária para embasar discussões no país.

*Professora-Doutora do Departamento de Tocoginecologia

Faculdade de Ciências Médicas, Universidade Estadual de Campinas (UNICAMP). 
Um estudo no Rio Grande do Sul encontrou uma incidência de 1/ 1000 nascidos vivos acometidos $^{6}$ e no Centro de Atenção Integral à Saúde da Mulher da Universidade Estadual de Campinas (CAISM-UNICAMP), a incidência foi 1,4/1000 antes da implementação de profilaxia vinculada a fatores de risco, de 1995 a 20007. Assim, a incidência estimada no Brasil, sem o uso de medidas profiláticas, pode ser inferior à dos EUA antes da implementação de medidas preventivas (2-3/ 1000), mas superior àquela que se alcançou com a adoção da cultura pré-natal $(0,2-0,4 / 1000)^{8}$. Também é superior aos valores observados no Reino Unido $(0,5 / 1000)$, onde se utilizam os fatores de risco como estratégia para orientar a profilaxia intra-parto ${ }^{9}$.

Pode ser que a diferença de sorotipos, além de características da população, influencie estes resultados. Há oito sorotipos do EGB, sendo o sorotipo III aquele que mais se observou entre os recém-nascidos doentes dos $\mathrm{EUA}^{10}$. No Brasil, Alves ${ }^{10}$ encontrou predomínio do sorotipo Ib, seguido por tipo II e Ia, entre gestantes de Jundiaí, São Paulo.

Beraldo et al. ${ }^{1}$ e Pogere et al. ${ }^{2}$ não encontraram nenhuma associação entre idade, paridade e escolaridade com colonização materna. Pogere et al. $^{2}$ observaram maior prevalência entre mulheres com mais de um parceiro sexual no último ano e maior freqüência de relações sexuais $(55,6 \%)$, uma associação que não tem sido consistentemente relatada na literatura. A impossibilidade de encontrar claros fatores de risco levou os autores a concluir que não é possivel selecionar, pelos antecedentes, as gestantes que devem receber profilaxia no momento do parto e a recomendar a utilização das culturas pré-natais de triagem. Também Pinto et al. ${ }^{11}$ não foram capazes de identificar fatores de risco em $46 \%$ dos casos de sepse neonatal por EGB.

Parece ser custo efetivo adotar/implementar a estratégia de triagem pré-natal se a incidência excede 1,2/1.000, o que seria 0,6/1.000 para a estratégia dos fatores de risco. Também se calculou que a incidência da sepse é 5,1/1000 partos quando a cultura é positiva e os fatores de risco são negativos e 0,9/1000 quando um dos fatores de risco é positivo e a cultura pré-natal é negativa, reforçando a idéia da maior efetividade de uma estratégia baseada em triagem pré-na$\operatorname{tal}^{3}$. Entretanto, a implementação da rotina de culturas no pré-natal entre a $35^{\mathrm{a}}$ e a $37^{\mathrm{a}}$ semanas certamente não é viável a curto ou médio prazo dentro da rede pública de assistência, na qual a urocultura não é exame recomendado numa rotina simplificada e o EGB não é incluído na rotina ampliada (Ministério da Saúde, 2001) ${ }^{12}$.

Além do problema óbvio de custo, os laboratórios nacionais, públicos e privados, não parecem estar preparados para atender a esta demanda da atenção perinatal. Há inúmeros detalhes, da coleta do material (sítio) à identificação do agente (características e conservação do meio de cultura), que precisam ser discutidos, normalizados e implementados. Nos EUA, tal proposta vinha sendo progressivamente implementada desde sua recomendação como estratégia opcional em 1996, permitindo-se encontrar 94\% de 207 laboratórios de sete estados realizando a coleta de amostras de vagina e reto, e 89\% usando meios seletivos enriquecidos em 2003 (CDC, 2004) ${ }^{13}$. Estas recomendações a respeito da cultura são respaldadas pela taxa de $50 \%$ de resultados falsos-negativos quando se usa meio não seletivo e amostra apenas vaginal ${ }^{3}$.

Alguns serviços, incluindo a maternidade da UNICAMP desde 2002, já têm adotado a profilaxia intra-parto baseada em fatores de risco. Também temos realizado estudos sobre a prevalência da colonização materna em situações de trabalho de parto prematuro e ruptura prematura de membranas, encontrando 25,2 e $34 \%$, respectivamente ${ }^{14}$. A realização da pesquisa nos auxiliou a estabelecer uma rotina laboratorial e as gestões para incluir a cultura na rotina pré-natal estão em andamento. Mesmo nos EUA, já se reconhece que é preciso simplificar e reduzir custos de procedimentos laboratoriais, mantendo a capacidade diagnóstica ${ }^{15}$.

Concluindo, a taxa de colonização materna, como mostraram estudos publicados nesta revista, e a incidência de complicações perinatais causadas pelo EGB sem o uso de intervenções profiláticas parecem ser similares ao que se observa na literatura internacional. Se a estratégia com cultura pré-natal não é viável a curto prazo, não se pode postergar a implementação diligente da estratégia por fatores de risco, em face da inquestionável eficácia da intervenção.

Paralelamente, precisamos criar um sistema de vigilância das causas de sepse neonatal e materna, para acompanhar as tendências da incidência e distribuição de agentes responsáveis. Quanto à introdução da cultura pré-natal, o maior conhecimento da situação nacional e do impacto das práticas implementadas em publicações de experiências nacionais e a discussão trans-disciplinar que inclua obstetras, neonatologistas, infectologistas, patologistas clínicos e gerentes de saúde deverão facilitar a decisão de conduta. 
De qualquer modo, no caso de partos anteriores à $35^{\mathrm{a}}$ semana (prévios à coleta da cultura) deve-se empregar profilaxia, assim como para os casos sem resultado disponivel ou colhidos há mais de cinco semanas do parto. Também é importante lembrar que não se recomenda, no momento, profilaxia no trabalho de parto se a cultura for negativa mesmo com fator de risco, e nos casos de cesáreas eletivas, sem trabalho de parto ou ruptura das membranas, mesmo se a cultura for positiva.

A maior certeza é que não se pode mais alegar ignorância, e que a implementação da profilaxia por fatores de risco é um passo necessário. Estamos no primeiro tempo deste jogo e a bola está nos pés dos obstetras!

\section{Referências}

1. Beraldo C, Brito ASJ, Saridakis HO, Matsuo T. Prevalência de colonização vaginal e anorretal por estreptococo do grupo B em gestantes de terceiro trimestre. Rev Bras Ginecol Obstet. 2004;26(7):543-9.

2. Pogere A, Zoccoli CM, Tobouti NR, Freitas PF, D`Acampora AJ, Zunino JN. Prevalência de colonização pelo estreptococo do grupo B em gestantes atendidas no ambulatório de pré-natal. Rev Bras Ginecol Obstet. 2005;27(4):172-8.

3. Schrag S, Gorwitz R, Fultz-Butts K, Schuchat A. Prevention of perinatal group B streptococcal disease. Revised guidelines from CDC. MMWR Recomm Rep. 2002;51(RR-11):1-22.

4. Dermer P, Lee C, Eggert J, Few B. A history of neonatal group B streptococcus with its related morbidity and mortality rates in United States. J Pediatr Nurs. 2004;19(5):357-63.

5. Associação Médica Brasileira. Projeto diretrizes. Assistência pré-natal [homepage na internet]. São Paulo; 2003 [citado em 10 ago 2003]. Disponivel em: http://www.amb.org.br

6. Miura E, Martin MC. Group B streptococcal neonatal infections in Rio Grande do Sul, Brazil. Rev Inst Med Trop Sao Paulo. 2001;43(5):243-6.

7. Calil R, Millen FC, Souza SN, Andrade EAPP, Marba STM. Doença por Streptococcus do grupo B: casuística da Unidade neonatal do CAISM/UNICAMP - 1995-2000. Anais? do $5^{\circ}$ Congresso Latino-Americano de Perinatologia; 2000 mês dias?; Rio de Janeiro, Brasil. Cidade: Editora; 2000. p. ?

8. Chen KT, Puopolo KM, Eichenwald EC, Onderdonk AB, Lieberman E. No increase in rates of early-onset neonatal sepsis by antibiotic-resistant group B Streptococcus in the era of intrapartum antibiotic prophylaxis. Am J Obstet Gynecol. 2005;192(4):1167-71.

9. Kenyon S, Blocklehurst P, Blackburn A, Taylor DJ. Antenatal screening and intrapartum management of Group B streptococcus in the UK. BJOG. 2004;111(3):226-30.

10.Alves VMN. Prevalência e fatores de risco associados à colonização retal e vaginal pelo Estreptococo do grupo B em parturientes e suas características fenotípicas [dissertação]. Campinas: Universidade de Campinas; 2005.

11. Pinto NM, Soskolne EI, Pearlman MD, Faix RG. Neonatal early-onset group B streptococcal disease in the era of intrapartum chemoprophylaxis: residual problems. J Perinatol. 2003;23(4):265-71.

12. Ministério da Saúde. Programa de humanização do pré-natal e do nascimento. Informações para gestores e técnicos. Brasília; 2001.

13.Centers for Disease Control and Prevention (CDC). Laboratory practices for prenatal group B streptococcal screening: seven states, 2003. MMWR Morb Mortal Wkly Rep. 2004;53(23):506-9.

14.Nomura ML. Colonização materna e neonatal por estreptococo do grupo B em gestantes com trabalho de parto prematuro e/ou ruptura prematura pré-termo das membranas [tese]. Campinas: Universidade de Campinas; 2005.

15.Rauen NC, Wesenberg EM, Cartwright CP. Comparison of selective and nonselective enrichment broth media for the detection of vaginal and anorectal colonization with group B streptococcus. Diagn Microbiol Infec Dis. 2005;51(1):9-12. 